\title{
Radio and Gamma-ray Observations of PSR J2043+2740
}

\author{
Aristeidis Noutsos \\ for the Fermi Collaboration \\ Max-Planck-Institut für Radioastronomie, Auf dem Hügel 69, 53121 Bonn, Germany \\ E-mail: anoutsos@mpifr-bonn.mpg.de
}

PSR J2043+2740 was first detected in gamma rays with the Fermi gamma-ray Space Telescope, in the first 6 months of the mission. It is one of the oldest, non-recycled gamma-ray pulsars known, and thus it gives us an insight into pulsars' ability to emit gamma rays as they age. We present gamma-ray data from 14 months of Fermi observations of PSR J2043+2740, which we have combined with recent radio-polarisation data and gamma-ray modelling to constrain the pulsar's emission geometry. Moreover, we have taken advantage of the large characteristic age of this pulsar to extend the studies of gamma-ray efficiency as a function of pulsar age. The efficiency of PSR J2043+2740 appears surprisingly low compared to pulsars of similar characteristic ages; we argue that this perhaps hints at a much smaller true age than the characteristic one. Last but not least, we discuss the possibility that PSR J2043+2740 is associated with the nearby Cygnus Loop and present the supporting and opposing evidence borne from our data analysis.

25th Texas Symposium on Relativistic Astrophysics - TEXAS 2010

December 06-10, 2010

Heidelberg, Germany 


\section{Introduction}

The Fermi Gamma-Ray Space Telescope was launched on 2008 June 11. During the first 6 months of the mission, $\gamma$-ray data from the main instrument on-board, the Large Area Telescope (LAT), were analysed for $\gamma$-ray pulsations from a preselected list of radio pulsars [15]. Amongst the pulsars that were detected that way was PSR J2043+2740, with a characteristic age $\tau_{\mathrm{c}}=P /(2 \dot{P})=1.2 \mathrm{Myr}$ - where $P$ and $\dot{P}$ are the spin period and spin-down rate, respectively. The above observations yielded the first, confident $\gamma$-ray detection for PSR J2043+2740 at the $5 \sigma$ level above the background [1].

PSR J2043+2740 was first discovered with Arecibo in a millisecond-pulsar survey at 430 MHz [17]. The $\gamma$-ray properties of PSR J2043+2740 make it a very interesting pulsar. It is one of the shortest-period, non-recycled (non-millisecond) $\gamma$-ray pulsars, without a birth-site association $(P=96.1 \mathrm{~ms})$. Also, PSR $\mathrm{J} 2043+2740$ is one of the oldest, non-recycled $\gamma$-ray pulsars, yet its spin-down lumonosity, $L_{\mathrm{sd}}=4 \pi^{2} I \dot{P} / P^{3}=5.6 \times 10^{34} \mathrm{erg} \mathrm{s}^{-1}$, where $I=10^{45} \mathrm{~g} \mathrm{~cm}^{2}$ is the neutron star's moment of inertia, is comparable to pulsars that are an order of magnitude younger: e.g. PSR J1835-0643. Last but not least, this pulsar's age and position arouses observational interest: its high characteristic age can be used to investigate claims for a correlation between $\gamma$-ray efficiency and age (see section 3.2) and its relative position to the Cygnus Loop begs the question of a possible association with the supernova remnant (see section 3.3 ).

The present document reports on the main results from our analysis of 14 months of Fermi data (2008 August 4 - 2009 October 17) from PSR J2043+2740. A more detailed description of this work can be found in [10].

\section{Data Analysis}

\subsection{Radio \& $\gamma$-ray Data}

We have used the Effelsberg 100-m radio telescope to obtain radio-polarisation data from PSR J2043+2740, at $1.4 \mathrm{GHz}$. The data were subjected to absolute polarisation calibration, which resulted in the integrated pulsar profile shown in fig. 1. By fitting a rotating vector model (RVM [11]) to the absolute polarisation position angles (PAs), we were able to determine the phase at the closest approach of our line-of-sight to the magnetic axis (fiducial phase). The best fit yielded $\phi_{0}=1.032 \pm 0.002$ corresponding to $\mathrm{PA}=17^{\circ} \pm 6^{\circ}$.

The $\gamma$-ray data from the Fermi LAT were subjected to cuts, whose purpose is to reduce the unwanted $\gamma$-ray background. After the cuts, the remaining 1,244 $\gamma$-ray events were phase-folded with a radio ephemeris obtained from the Jodrell Bank pulsar-timing database [4], covering the period from 2008 June 17 to 2009 October 17; the total lightcurve above $0.1 \mathrm{GeV}$, as well as the lightcurves between 0.1 and $0.3 \mathrm{GeV}, 0.3$ and $1 \mathrm{GeV}$, and above $1 \mathrm{GeV}$ are shown in fig. 2 . The significance of the detection above $0.1 \mathrm{GeV}$ was $\approx 7 \sigma$. The characterisation of this pulsar's lightcurve remains, as in the original detection, a double-peaked lightcurve. The phase separation of the two $\gamma$-ray peaks is $\Delta=0.35 \pm 0.04$, and the minimum radio- $\gamma$ ray phase lag is $\delta=0.2 \pm 0.03$; these values are consistent with those published in the first Fermi pulsar catalog [1]. 


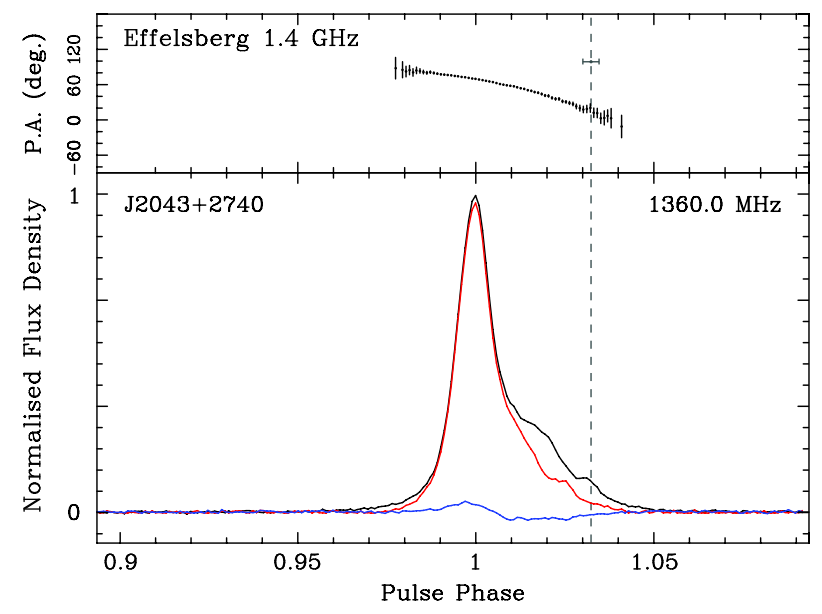

Figure 1: Absolute polarisation profile of PSR J2043+2740. The black solid line corresponds to the total intensity across the pulse period, the red line, to the linearly polarised intensity, and the blue line, to the circularly polarised intensity. The top panel shows the absolute polarisation-position-angle profile across the pulse.

\subsection{Spectral Analysis}

We obtained the phase-averaged $\gamma$-ray spectrum of PSR J2043+2740 above $0.1 \mathrm{GeV}$. The spectral analysis was performed using the Fermi Science Tools and included all sources within $15^{\circ}$ of the pulsar, taken from the 11-month Fermi catalog of $\gamma$-ray sources [2]. The emission was modelled as a simple power law with an exponentially cut-off high-energy tail of the form $d N / d E=N_{0}(E / 1 \mathrm{GeV})^{-\Gamma} \exp \left(-E / E_{\mathrm{c}}\right)$. In this expression, $\Gamma$ is the power-law index, $E_{\mathrm{c}}$ is the cut-off energy of the exponential cut-off and $N_{0}$ is a prefactor term. The best-fit values were $\Gamma=$ $1.1 \pm 0.2$ and $E_{\mathrm{c}}=0.8 \pm 0.1 \mathrm{GeV}$. Integration of the spectrum above $0.1 \mathrm{GeV}$ gives an energy flux of $G_{>0.1 \mathrm{GeV}}=(1.5 \pm 0.2) \times 10^{-11} \mathrm{erg} \mathrm{cm}^{-2} \mathrm{~s}^{-1}$. These values are in agreement with [1], but with improved statistics. It should be noted that the derived spectrum favours a high-altitude-emission origin, such as described by the Outer Gap (OG) and Two Pole Caustic (TPC) models .

\section{Discussion}

\subsection{Emission Geometry}

The RVM fits to the radio-polarisation data were also used to derive confidence contours on the sky-projected orientation of the magnetic and spin axes of PSR J2043+2740, with respect to our line of sight. The greyscale contours of fig. 3 represent the 1,3 and $5 \sigma$ confidence contours for the magnetic inclination, $\alpha$, and the viewing angle, $\zeta$. In order to better constrain the emission geometry, we combined the radio-polarisation contours with $\gamma$-ray constraints for this pulsar, based on the recent ATLAS maps of simulated $\gamma$-ray profiles by [18]. Fig. 3 shows the radio and the $\gamma$-ray (magenta and green) contours superimposed, for the OG and TPC models, and for the lightcurve properties of PSR J2043+2740. The overlapping regions of all contours (outlined in the figure with solid lines) show the valid ranges of $\alpha$ and $\zeta$ consistent with all our data. For the TPC model, we 
found that $\alpha \sim 52^{\circ}-57^{\circ}$ and $\zeta \sim 61^{\circ}-68^{\circ}$ are good parameter ranges. For the OG geometry, there were two discrete parameter ranges: (a) $\alpha \sim 62^{\circ}-73^{\circ}$ and $\zeta \sim 74^{\circ}-81^{\circ}$ and (b) $\alpha \sim 72^{\circ}-83^{\circ}$ and $\zeta \sim 60^{\circ}-75^{\circ}$.

Since both models were consistent with the radio and $\gamma$-ray data, we cannot exclude either model based on the contour maps. However, we note that the two peaks in the $\gamma$-ray lightcurve of PSR J2043+2740 appear to mirror each other, with the outer edges having a steeper fall-off than the inner edges. This is the expected profile from an OG model and is less consistent with the expected shape from a TPC model (see Appendix in [18]). Therefore, we conclude that, for the case of PSR $\mathbf{J} 2043+2740$, the OG model is favoured.

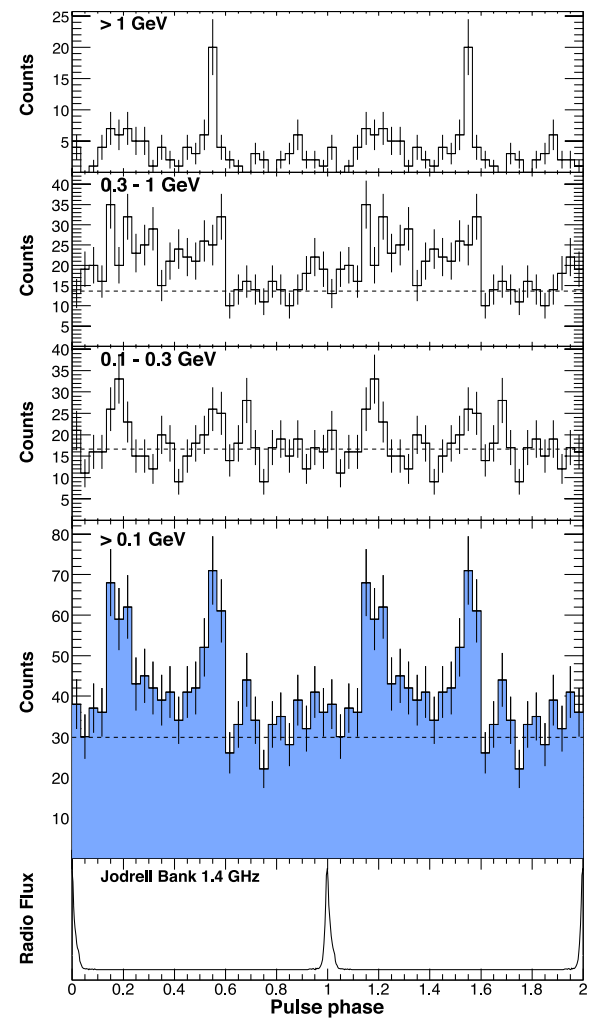

Figure 2: $\gamma$-ray lightcurves of PSR J2043+2740 above $0.1 \mathrm{GeV}$ (blue-shaded histogram). The bottom panel shows the radio profile of PSR J2043+2740 from Jodrell Bank data; phase 0 of the $\gamma$-ray data corresponds to the maximum of the radio emission.

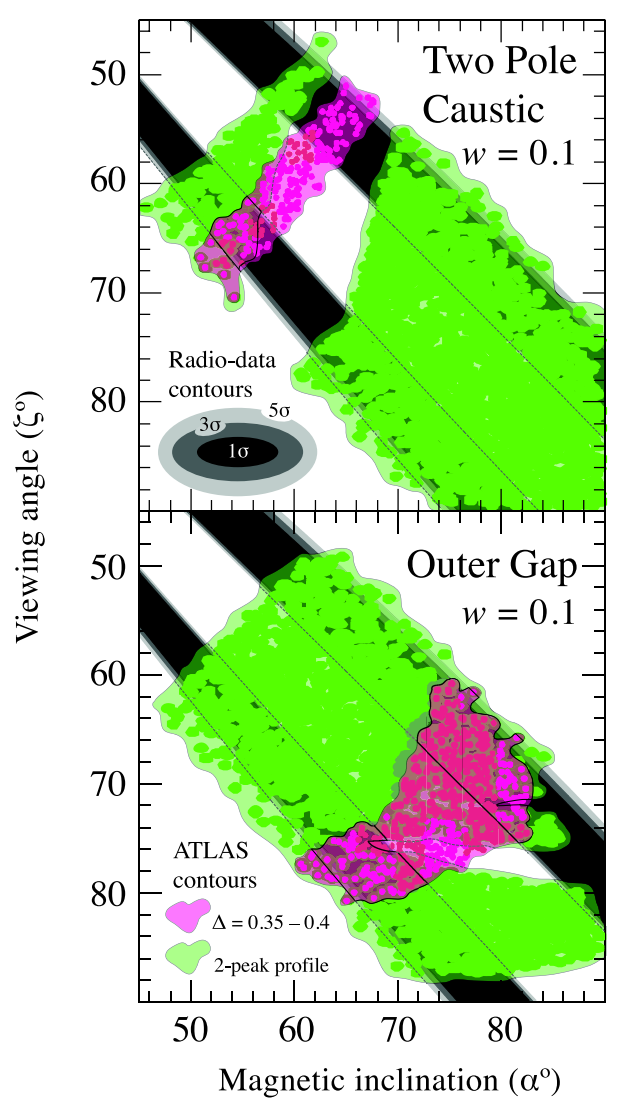

Figure 3: Joint-probability contours of magnetic inclination $(\alpha)$ and viewing angle $(\zeta)$, from our radiopolarisation analysis (greyscale contours) and the ATLAS of $\gamma$-ray lightcurves corresponding to the properties of PSR J2043+2740 (green and magenta contours).

\section{$3.2 \gamma$-ray Efficiency}

The $\gamma$-ray luminosity of a pulsar is defined as $L_{\gamma}=4 \pi f_{\Omega} G_{>0.1 \mathrm{GeV}} D^{2}$, where $f_{\Omega}$ is a fluxcorrection factor, correcting for the degree of anisotropy of the phase-averaged pulsar flux over the whole sky [18]. Based on the $\gamma$-ray luminosities of a number of non-recycled $\gamma$-ray pulsars 
with available distances, [1] calculated the $\gamma$-ray efficiencies, $\eta=L_{\gamma} / L_{\mathrm{sd}}$, under the assumption of $f_{\Omega}=1$.

The high characteristic age of PSR J2043+2740 is a good opportunity to review the plots of $\eta$ against $\tau_{\mathrm{c}}$, which earlier work - having significantly fewer available measurements - suggested a correlation between those quantities (see e.g. [19]). Especially with the addition of PSR $\mathrm{J} 2043+2749$, the characteristic-age range in those studies is extended by an order of magnitude compared to the rest of the non-recycled, $\gamma$-ray pulsars with measured efficiencies. A scatter plot of $\eta$ versus $\tau_{c}$ is shown in fig. 4. It is clear from this plot, despite the large error bars, that there is an evident correlation between $\eta$ and $\tau_{\mathrm{c}}$, with older pulsars appearing more efficient in producing $\gamma$ rays than younger ones.

\subsection{Birthplace, Orientation and Proper Motion}

The Cygnus Loop region, shown in fig. 5, is a complex of two supernova remnants, G74.3-8.4 and G72.9-9.0 [16]. This remnant has already been searched by a number of authors for the central compact source, but so far there has not been conclusive evidence for an association [17][12][9]. PSR J2043+2740 lies near the south-western edge of G72.9-9.0, which perhaps suggests an association between the two objects. However, the present evidence is not in favour: the distance to the remnant has been estimated to $D=540_{-80}^{+100} \mathrm{pc}$ [3]; but based on the pulsar's dispersion measure $\left(\mathrm{DM}=21.0 \pm 0.1 \mathrm{pc} \mathrm{cm}^{-3}\right)$, and assuming the NE2001 Galactic electron-density model, the pulsar distance is much larger: i.e. $D=1.8 \mathrm{kpc}$. In addition, the pulsar's characteristic age is two orders of magnitude higher than the remnant's: the latter has been estimated to $<12 \mathrm{kyr}$ [14]. Last but not least, given the remnant's age and observable limits, an association would imply that the pulsar had been travelling at $>980 \mathrm{~km} \mathrm{~s}^{-1}$. Such transverse velocity is significantly higher than the average measured for the sample of known pulsars, i.e. $\approx 400 \mathrm{~km} \mathrm{~s}^{-1}[5]$.

Contrary to the above arguments, the position of PSR J2043+2740 in the plot of fig. 4 is somewhat removed from the general trend suggested by the rest of the sample. If the pulsar was born in the Cygnus Loop, then its age would necessarily be $\tau=12 \mathrm{kyr}$ and its distance, $D=540 \mathrm{pc}$. Under this assumption, the new position of PSR J2043+2740 in that plot (represented with a solid, grey square) appears more consistent with the overall trend. Therefore, based on $\gamma$-ray energetics and the observed general trend in $\tau_{\mathrm{c}}-\eta$, there is some support for an association.

We have tried to investigate whether the proper motion of PSR J2043+2740 can give clues as to the pulsar's origin: a velocity vector pointing away from the centre of G72.9-9.0 $0^{1}$ would provide good support for an association. At the moment, there are no available direct, VLBI measurements of this pulsar's proper motion; therefore, our first approach was to use pulsar timing to fit for a proper motion. However, PSR J2043+2740 possesses a large amount of long-term ('red') timing noise $\left(\ddot{v}=56.04 \pm 0.57 \times 10^{-24} \mathrm{~s}^{-3}\right)$, which in fact ranks it amongs the pulsars with the highest noise observed (compare with Table 1 of [6]). Before a proper motion can be estimated, this noise needs to be modelled and removed. Unfortunately, due to the significant magnitude of the red noise, we were unable to completely account for it, which compromised the proper-motion estimate.

Alternatively, we tried to use the pulsar's orientation in space, derived from our polarisation measurements. It has been claimed that there is strong evidence for a correlation between the sky-

\footnotetext{
${ }^{1}$ The required velocity for an association with G74.3-8.4 is much too high and therefore improbable.
} 
projected spin-axis orientations of young pulsars and their velocity vectors [7]. If the spin-velocity alignment scenario is true for PSR J2043+2740 and if the pulsar was born in the Cygnus Loop, then we expect that the derived spin-axis orientation points, within the errors, towards the remnant. Our polarisation measurements show that the fiducial PA of the magnetic axis, which corresponds to the PA of the spin axis, has a position angle of $17^{\circ}$ north through east; this direction is shown in fig. 5. It is evident from the figure that the derived orientation is significantly offset $\left(=19^{\circ} \pm 6^{\circ}\right)$ from the direction of G72.9-9.0.

\section{Conclusions}

Our polarisation measurements combined with the spin-velocity alignement hypothesis do not favour the association between PSR J2043+2740 and the Cygnus Loop. It is true that there is a significant spread of offsets between the spin axes and the proper motion vectors in the work of [7]. However, roughly $90 \%$ of the pulsars in that analysis have smaller offsets than our measured value, which makes the association on those grounds very unlikely.

Recently, it was proposed that a large part of the pulsar timing noise can be attributed to pulseshape variations correlated with changes in $\dot{P}$ [8]. PSR J2043+2740 is a good example of such behaviour, exhibiting changes of a $100 \%$ in the pulse's FWHM over timescales of 200 days. In the near future, we will be able to fit out these variations, which will allow us to reliably estimate this pulsar's, as well as other noisy pulsars' proper motions.

Lastly, a direct measurement of the proper motion of PSR J2043+2740 with VLBI would certainly provide the most conclusive arguments for the pulsar's connection to the Cygnus Loop. If the pulsar was born in the centre of G72.9-9.0, then the required proper motion that would lead

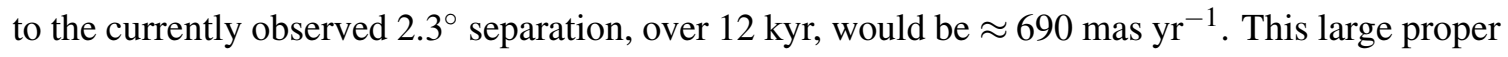
motion would be easily measureable with VLBI.

In conclusion, the connection of PSR J2043+2740 to the Cygnus Loop is still likely to remain an open question, until either a future VLBI campaign or improvements in pulsar timing reveal this pulsar's proper motion.

\section{Acknowledgements}

The Fermi LAT Collaboration acknowledges support from a number of agencies and institutes for both development and the operation of the LAT as well as scientific data analysis. These include NASA and DOE in the United States, CEA/Irfu and IN2P3/CNRS in France, ASI and INFN in Italy, MEXT, KEK, and JAXA in Japan, and the K. A. Wallenberg Foundation, the Swedish Research Council and the National Space Board in Sweden. Additional support from INAF in Italy and CNES in France for science analysis during the operations phase is also gratefully acknowledged.

\section{References}

[1] Abdo, A. A., et al. 2010, ApJ, 713, 154

[2] Abdo, A. A., et al. 2010, ApJ, 188, 405

[3] Blair, W. P., Sankrit, R., Torres, S. I., Chayer, P., \& Danforth, C. W. 2009, ApJ, 692, 335 


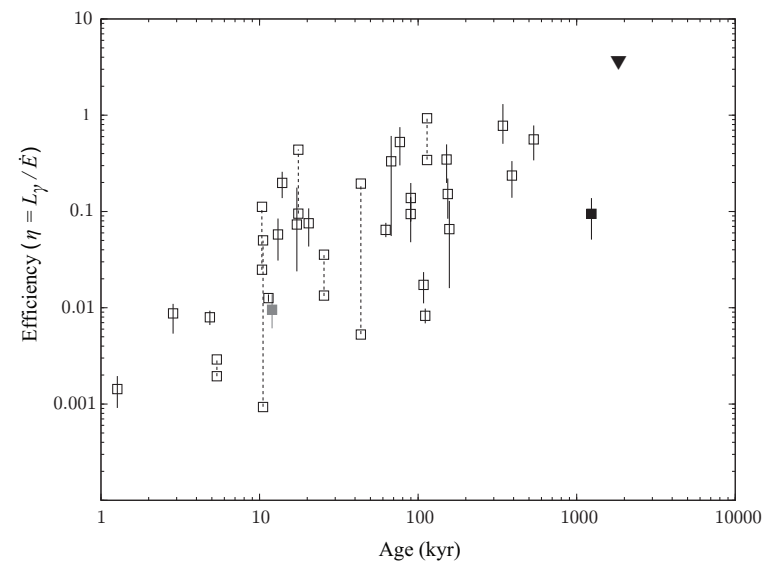

Figure 4: Scatter plot of $\gamma$-ray efficiency versus characteristic age for all known, non-recycled $\gamma$-ray pulsars with available distance estimates (empty squares). The filled square corrsponds to PSR J2043+2740 and the solid triangle denotes an upper limit for PSR J1836+5925. The light-grey square corresponds to the efficiency and age of PSR J2043+2740, assuming the pulsar was born in the Cygnus Loop. For 5 pulsars, there are two distance estimates: for those, the plot presents two possible values connected with a dashed line.

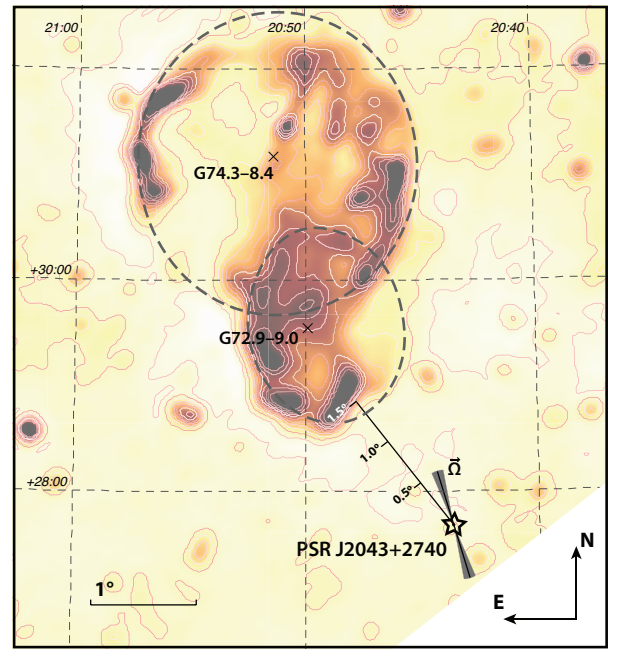

Figure 5: Radio map of the Cygnus Loop region from $1.4 \mathrm{GHz}$ data from the Effelsberg Medium Latitude Survey (EMLS; [13]). The position of PSR J2043+2740 is shown with a star symbol. The derived orientation of the spin axis is shown at the pulsar's position with a grey-shaded wedge. The boundaries of G74.3-8.4 (uppermost) and G72.9-9.0 (lowermost) are delineated with dashed ovals, with the crosses marking the geometrical centres of the two remnants.

[4] Hobbs, G., Lyne, A. G., Kramer,M., Martin, C. E., \& Jordan, C. 2004, MNRAS, 353, 1311

[5] Hobbs, G., Lorimer, D. R., Lyne, A. G., \& Kramer, M. 2005, MNRAS, 360, 974

[6] Hobbs, G., Lyne, A. G., \& Kramer, M. 2010, MNRAS, 402, 1027

[7] Johnston, S., et al. 2005, MNRAS, 364, 1397

[8] Lyne, A., Hobbs, G., Kramer, M., Stairs, I., \& Stappers, B. 2010, Science, 329, 408

[9] Miyata, E., et al. 1998, PASJ, 50, 475

[10] Noutsos, A., et al. 2011, ApJ, 728, 77

[11] Radhakrishnan, V., \& Cooke, D. J. 1969, Astrophys. Lett., 3, 225

[12] Ray, P. S., et al., 1996, ApJ, 470, 1103

[13] Reich,W., et al. 2004, in The Magnetized Interstellar Medium, ed. B. Uyaniker,W. Reich, \& R. Wielebinski (Katlenburg-Lindau: Copernicus GmbH), 45

[14] Sankrit, R., \& Blair, W. P. 2002, ApJ, 565, 297

[15] Smith, D. A., et al. 2008, A\&A, 492, 923

[16] Sun, X. H., Reich,W., Han, J. L., Reich, P., \& Wielebinski, R. 2006, A\&A, 447, 937

[17] Thorsett, S. E., Ray, P. S., Kulkarni, S. R., \& Prince, T. A. 1994, IAU Circ., 6012

[18] Watters, K. P., Romani, R. W., Weltevrede, P., \& Johnston, S. 2009, ApJ, 695, 1289

[19] Zhang, L., Cheng, K. S. 1998, A\&A, 335, 234 